\title{
Two puzzles about requirements
}

\author{
Rick Nouwen \\ Utrechts Instituut voor Linguïstiek (Universiteit Utrecht)
}

\begin{abstract}
I discuss the semantics of statements of minimum and maximum requirement. I show that, on standard assumptions, such statements receive a non-sensical interpretation. I solve this puzzle by pointing out certain semantic features shared by all end-point markers akin to minimum and maximum.
\end{abstract}

\section{First puzzle: Minimum requirements}

My friend and me are having a bet in which I claim to be able to score at least 300 points in the game of scrabble we are about to start. The following would be an accurate paraphrase for the bet in question.

(1) The minimum number of points I need to score to win the bet is 300 .

That is, the bet involves a minimum requirement: If I score 300, I win. If I score more, I also win. But if I score less, then I lose. To be sure, at first sight it seems obvious why (1) is interpreted as such, for I do need to score 300 , and 300 is the minimum score that makes me win the bet. Yet, when we make things precise, and given common assumptions on the semantics of modal auxiliaries, it turns out that it is rather mysterious why (1) means what it means.

The common assumptions I am alluding to are, first of all, that need is a universal quantifier over possible worlds and, second, that the to-phrase in (1) restricts quantification over possible worlds (as in von Fintel and Iatridou's [2]). In other words, "to $p$, need to $q$ " is true if and only if all the $p$-worlds are $q$-worlds; i.e. if $p$ entails $q$. At first sight, this view appears to make good predictions. For example, a case like (2) is now interpreted as saying that you went to the Twijnstraat in all the worlds where you got good cheese.

(2) To get good cheese, you need to go to the Twijnstraat.

However, when we apply the above assumptions to (1), the outcome is very puzzling. Note first the following: in the scenario I sketched about the scrabble bet, there are no worlds in which I win the bet while scoring fewer than 300 points. Furthermore, the worlds where I do win come in many variations: in some (but not all) of them I score 300 points, in some (but not all) my score is 301 , in some (but not all) it is 302,310 , or even 550 . The problem is that the most obvious referent for the minimum number of points I need to score is the smallest number $x$ such that $I$ scored $x$ points is true in all relevant worlds. However, for no value for $x$ is this open sentence true in all bet-winning worlds. 


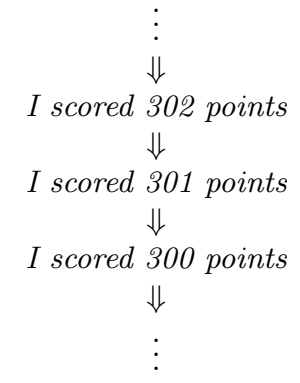

Fig. 1. The at least interpretation for numerals

In other words, one would expect the definite description the minimum number of points I need to score to fail to refer in the described situation.

It might appear that there is an obvious solution. If we assume that numerals are interpreted as lower-bounded only (usually, this is dubbed the at least interpretation for numerals), then we get an entailment scale as in figure 1. Given this scale, it is now true that in all bet-winning worlds I scored 300 points, for the worlds where my exact score was higher will be worlds in which I scored at least 300 points. Unfortunately, if we assume that such an entailment scale is appropriate then, by entailment, it is also true that in all bet-winning worlds I scored 200 points. In fact, it is entailed that I score a single point in every bet-winning world. Consequently, the minimum number of points I need to score to win the bet is now predicted to be 1 , not 300. Yet, (3), obviously, seems an unacceptable way of paraphrasing the bet between my friend and me.

(3) The minimum number of points I need to score to win the bet is 1 .

In sum, independent of how we interpret numerals, it appears far from straightforward how to come to a compositional interpretation of (1).

\section{Second puzzle: Maximum requirements}

If we assume the entailment scale in figure 1, then there is a further puzzle. The proposition I scored 1 point is true in all bet-winning worlds, and so is the proposition I scored 300 points. The proposition I scored 301 points, however, is the first proposition in the scale that is not true in all bet-winning worlds. (It's only true in some.) This makes 300 the highest value for $x$ such that $I$ scored $x$ points is true in all victorious worlds and so we predict that instead of (1), the correct way of paraphrasing the bet is, in fact, (4). Clearly, this is an unwelcome prediction, for the scenario I sketched was a prime example of what we call minimum requirements, not of maximum ones.

(4) The maximum number of points I need to score to win the bet is 300 . 
In the given scenario there was no upper limit on my score. That is, the bet is only about me scoring 300 or more. No matter how much higher than $300 \mathrm{I}$ score, I will keep on winning. In certain other scenarios maximal requirements do make sense, however. Take the following example.

(5) The maximum number of sets needed to decide a men's tennis match is 5 .

In men's tennis, the first player to win three sets wins, hence (5). At the same time, this means that there can be no men's tennis match which lasts for fewer than 3 sets. In other words:

(6) The minimum number of sets needed to decide a men's tennis match is 3 .

Once again, we derive the wrong interpretation for such examples under the standard assumptions discussed above. Let us first assume that numerals create entailment scales, as in figure 1. That is, if 4 sets were played in a match, then this entails that 3 sets were played, as well as that 2 sets were played, etc. The minimum number $n$ of sets such that $n$ sets are played in every world in which the match is decided is now 1 . The maximum number $n$ of sets such that $n$ sets are played in every match-deciding world is 3 , since all such worlds contain (at least) 3 sets, while only some contain a 4 th or a 5 th one.

Things are no better if we interpret numerals as doubly-bound. In that case the definite descriptions in (5) and (6) will fail to refer, for there exists no $n$ such that in all possible ways in which a tennis match could be decided the match counted exactly $n$ sets.

\section{Modal Force}

The two puzzles above point out a problem with the interaction between universal modals and scalar operations. Abstracting away from the scenarios above, the puzzles can be generalised as follows. Let $\mathcal{P}$ be a scale of propositions, as in (7).

$$
p_{1}<p_{2}<p_{3}<\ldots<p_{i}<p_{i+1}<\ldots
$$

Following standard assumptions, a proposition $p_{k}$ is a minimum requirement for $q$ if it is the case that $p_{k}$ is entailed by $q$, while for no $l<k, p_{l}$ is entailed by $q$. If (7) is an entailment scale, however, then for any $p_{k}$ it holds that $p_{k} \models p_{k-1}$ and, in fact, $p_{k} \models p_{1}$. So, if $q$ entails $p_{k}$, then it also entails $p_{1}$. In other words, the minimum requirement for $q$ could only ever be $p_{1}$, which renders the notion of minimum requirement quite useless. If, however, $\mathcal{P}$ is not an entailment scale, then it is easy to imagine that $p_{k}$ is entailed by $q$ while this is not the case for any proposition that is lower on the scale. Unfortunately, this is not how we normally understand the notion of minimum requirement. In fact, part of the problem with our assumed definition of minimum requirement is that if $p_{k}$ is a 
minimum requirement for $q$, then $q$ need not entail $p_{k}$. For instance, if my bet is that I will score 300 or more in the game of scrabble, then me winning the bet does not entail me scoring exactly 300 points. ${ }^{1}$

There is a surprising way out: minimal and maximal requirements are not about necessity, but rather about possibility. Assume $\mathcal{P}$ to be a non-entailment scale: $p_{k}$ is a minimal (maximal) requirement for $q$ iff $p_{k}$ is compatible with $q$ and there is no $l<k(l>k)$ such that $p_{l}$ is compatible with $q$. In the scrabble bet scenario, the proposition that I scored exactly 300 points is the lowest ranked proposition such that there exists a bet-winning world in which that proposition is true. Furthermore, 3 is the smallest number $n$ such that there is a world in which the tennis match is decided in such a way that the number of played sets is exactly $n$. Also, 5 is the highest number $n$ such that there is a world in which the match is decided such that the number of played sets is exactly $n$. Thus, under the assumption that the modal force of need is existential, the analysis of examples like (1), (5) and (6) appears straightforward.

There is however clear evidence that the modal force of need and it's kin is not existential. For instance, if it were, we would predict (8) to be true. The intuition, however, is that it is false.

(8) To decide a men's tennis match, you have to play exactly 3 sets.

Below, I will argue that although the semantics of need and its kin involves universal quantification, operators like minimum and maximum are special in that they tend to force a weaker interpretation of their complement.

Before I provide the details of this analysis and give motivation that favours it, I will first briefly say a bit more about the data. Central to the puzzles that I presented above is a rather specific family of noun phrases, namely definite descriptions that contain a minimality or maximality operator and a necessity modal. It is important to note that the specific modal or the specific scalar operator does not matter. First of all, statements of minimal or maximal requirement

${ }^{1}$ Connected to this discussion is the following. Von Fintel and Iatridou [2] observe that their theory predicts that (i-a) entails (i-b).

(i) a. To get good cheese, you have to go to the Twijnstraat.

b. $\Rightarrow$ To get good cheese, you have to breathe.

If you go to the Twijnstraat in all worlds in which you get good cheese, then since you breathe in all the worlds in which you go to the Twijnstraat, it follows that you breathe in all worlds in which you get good cheese. Von Fintel and Iatridou judge (i-b) true, yet unhelpful in the context of (i-a), an intuition I agree with. This means that even though the data in (i) might be slightly unintuitive at first sight, von Fintel and Iatridou's theory appears on the right track in predicting that there is an entailment here. However, the truth of the following example, suggested to me by David Beaver, is a further prediction of the theory, but it is not clear that it is a welcome one.

(ii) To climb Everest you need 3 to equal $2+1$. 
are not limited to the modal to need. Must, require, should and have to allow for similar constructions, as in the following naturally occurring examples, and pose exactly the same puzzles.

(9) Determine the smallest number of digits that must be removed from $\mathrm{x}$ so that the remaining digits can be rearranged to form a palindrome.

(10) REM level is the minimum number of BYTES you require to continue.

(11) What is the minimum number of karanga I should know before I can say that I can karanga?

(12) We are usually interested in knowing the smallest number of colors that have to be used to color a graph.

(13) What is the shortest distance one needs to travel to visit all 30 teams in 28 major league cities?

Note too that expressing minimal (or maximal) requirements is not limited to the expressions minimum and maximum (and their derivations). The phenomenon extends to using superlatives (like smallest in (9) and (12), or shortest in (13)).

\section{Towards an analysis: restricting the modal base}

Above, I argued that despite the puzzles central to this work, modals like need are best analysed as universal quantifiers. They just appear to have existential modal force whenever they interact with expressions of minimality/maximality. This is in a rather specific sense reminiscent of the discussion of modals in Lillooet Salish by Rullmann et al. [7], for they argue that in that language modals have variable quantificational force, with universal force being the default. ${ }^{2}$ Their analysis is based on an idea originating from Klinedinst [4]. Klinedinst proposes that modals are distributivity operators with respect to a plurality of worlds. According to this proposal modal force is not a matter of existential versus universal quantification over possible worlds, but rather a matter of whether the modal base is definite (necessity) or indefinite (possibility). ${ }^{3}$ Put differently, whereas necessity modals quantify distributively over the whole modal base, possibility modals quantify distributively over just a subset of modal base worlds.

\footnotetext{
${ }^{2}$ A different possible route to follow would have been to connect the puzzles of minimum and maximum requirement to Schwager's observations in [8]. She discusses the fact that necessity modals are sometimes compatible with anti-exhaustifiers like for example, resulting in a weakening of the modal force. I leave it to further research to investigate whether Schwager's observations are in any deeper way linked to the puzzles discussed above.

${ }^{3}$ I should note, however, that the semantics of minimum/maximum requirement does not turn out to be solely due to the interaction between minimality/maximality operators and definiteness. There is no clear parallel between minimum requirements and cases where minimality scopes over a definite noun phrase. For instance, it appears impossible to interpret (i) as saying that the man who married the youngest woman married a woman of 20 years old.
} 
Rullmann et al. implement Klinedinst's idea by providing the general scheme for the interpretation of modal expressions in (14).

$$
\begin{aligned}
\llbracket \operatorname{MODAL}(f)(w)(\varphi) \rrbracket= & \forall w^{\prime} \in f(\text { modal_base }(w)): w^{\prime} \in \varphi \\
& \text { where } f \text { maps a set of worlds to one of its subsets }
\end{aligned}
$$

If $f$ is the identity function, then the scheme represents an operator which universally quantifies over the worlds in the modal base, which is equivalent to the standard interpretation of necessity modals. If $f$ is existentially closed, thus allowing quantification over a proper subset of the modal base, then the scheme represents a possibility modal. Rullmann et al. suggest that in Lillooet salish, $f$ is not specified for modal expressions, which is why these expressions have variable modal force. In English, expressions like need fix $f$ to be the identity function.

Obviously, a view on modals along these lines opens up the theoretical possibility for variable quantificational force for English models too. Say, we interpret need as follows, where the underlined conjunct $\exists f[f=\lambda W . W]$ is interpreted with widest possible scope (akin a presupposition). (I'm assuming a dynamic interpretation of $\exists$ ).

$$
\llbracket \text { need } \rrbracket=\lambda p \cdot \lambda w \cdot \exists f[f=\lambda W \cdot W] \& \forall w^{\prime} \in f(\text { modal_base }(w)): w^{\prime} \in p
$$

The choice of the identity function for determining the domain of quantification can be overruled by existential quantification over $f$ with narrower scope. As I will suggest below, scalar operators like minimum/maximum introduce existential quantifiers in their scope. It is this fact that weakens the modal force of necessity modals in the scope of such operators. For instance, example (16) is interpreted as (17), where the first conjunct is rendered vacuous by the local re-quantification over $f$.

(16) The minimum number of points I need to score is 300 .

$$
\begin{aligned}
& \exists f[f=\lambda W . W] \& \\
& \min \left(\lambda n . \exists f\left[\forall w^{\prime} \in f(\lambda w . \text { I win the bet in } w): \text { I score } n \text { points in } w^{\prime}\right]\right)=300
\end{aligned}
$$

This says that there exists a subset of worlds in which I win the bet such that I score exactly 300 points in every world in that subset and that there is no subset of the set of bet-winning worlds such that for each world in that set I score $n<300$ points.

The mechanism with which we derive (17) (i.e. existential closure in the scope of the minimality operator) might seem far-fetched, but as I will show next, it turns out that there is a general case to be made that scalar end-point markers, like superlatives and expressions like minimum and maximum, introduce existential structures in their scope. To be able to show this I will need modified numerals to enter into the discussion.

(i) (Five older men get married in the same year, all to significantly younger spouses.) The youngest woman the men married is 20 years old. 


\section{$5 \quad$ Modified Numerals}

So far, I have been assuming that, in statements of minimum or maximum requirement, the scope of minimum and maximum is wider than that of the modal. So, I have been analysing (1) as (18-a), rather than (18-b). ${ }^{4}$

(1) The minimum number of points I need to score to win the bet is 300 .

$$
\begin{aligned}
& \text { a. } \quad \min _{n}(\square[\text { I score } n \text { points }])=300 \\
& \text { b. } \quad \square\left[\min _{n}(\text { I score } n \text { points })=300\right]
\end{aligned}
$$

Note that an analysis along the lines of (18-b) does not solve our puzzles. On an 'exactly' reading for $n$ points, there is just the single value for $n$ which makes I score $n$ points true in a specific world. The use of minimally would then be vacuous. Moreover, (18-b) is true if and only if I win the bet providing I score exactly 300 points, no more, no fewer. This is not a minimum requirement. Worse still, on an analysis along the lines of (18-b), we would expect that there is no difference between (1) and (19).

The maximum number of points I need to score to win the bet is 300 .

On an at least perspective for $n$ points, (18-b) will be a contradiction. Since for any $n, I$ score $n$ points entails that I scored a single point, (18-b) ends up stating that $\square[1=300]$.

Interestingly, there is a variation on (18-b) that yields the correct truthconditions without the need for a change in modal force for need.

$$
\square\left[\iota_{n}(\text { I score } n \text { points }) \geq 300\right]
$$

This analysis is not as far fetched as it might seem at first sight. As a numeral modifier, minimally shares its semantics with at least. In other words, the proper treatment of (1) could be thought to be whatever works for (21) or (22).

(21) To win the bet, I need to score minimally 300 points.

(22) To win the bet, I need to score at least 300 points.

\footnotetext{
${ }^{4}$ It is difficult to extend the above puzzles of minimal and maximal requirement to cases of epistemic modality. This might actually be expected if the analysis of a wide scope minimality operator is on the right track, given the generalisation that epistemic modals tend to take wide scope (cf. [1]).

Consider the following example. Say, you have seen me put 10 marbles in a box, but you do not know how many marbles there were in the box to begin with. Structurally, your knowledge state now resembles that of a minimal requirement scenario: in all compatible worlds, there are (at least) 10 marbles in the box, while in no compatible worlds there are fewer than 10 marbles in the box. Yet, in contrast to the examples given above, we cannot express this knowledge state as (i).
}

(i) ??The minimum number of marbles that must be in the box is 10 
Unfortunately, there are reasons to believe that (20) is too simplistic as an analysis for (21) or (22). As Geurts and Nouwen [3] argue in detail, at least does not correspond to the $\geq$-relation. Moreover, I recently showed that both minimally and at least are part of a class of numeral modifiers that is incompatible with specific amounts [6]. That is, whereas (23) is felicitous and true, (24) is unacceptable.

(23) A hexagon has more than 2 sides.

(24) \#A hexagon has $\{$ at least / minimally $\} 2$ sides.

If minimally corresponded to $\geq$, then it is a mystery why (24) is infelicitous, for it ends up stating that $6 \geq 2$, which is simply a true proposition. Note also, that one cannot argue that (24) is true but underinformative and therefore infelicitous, for the same argument would wrongly predict (23) to be infelicitous, since this states the true and underinformative $6>2$. The conclusion I draw from this in [6] is that, unlike the comparative more than, modifiers like minimally are not operators that compare two values. For our present purposes this means that (20) will not do as an analysis of (21) or (22), and that it therefore seems unlikely that the solution to the minimum requirement puzzle lies in interpreting minimum as a (reconstructed) narrow scope $\geq$-relation.

A further property of numeral modifiers like minimally is that they trigger readings of speaker uncertainty (cf. [3], [5], [6]). For instance, (25) is interpreted as being about the minimum number of people John might have invited (according to the speaker).

John invited \{ minimally / at least $\} 30$ people to his party. (\#To be precise, he invited 43.)

Such speaker uncertainty readings carry over to adjectives like minimum. ${ }^{5}$

The $\{$ minimum / smallest $\}$ number of people John invited to the party is 30 .

(\#To be precise, it's 43.)

Apart from understanding (26) as a case of speaker uncertainty, one might also understand it as saying that 30 is the smallest number of people that John, at some time in the past, invited to the party. Crucially, all available readings somehow involve existential quantification.

The point I want to make is that there is a general puzzle underlying the interaction of universal modals and scalar operators, be they adjectives like minimum, smallest, highest etc. or numeral modifiers like minimally and at least. ${ }^{6}$

\footnotetext{
${ }^{5}$ I am grateful to an anonymous Amsterdam Colloquium reviewer for urging me to attend to the relevance of such data.

${ }^{6}$ In fact, an anonymous reviewer suggests that the data extends to cases where minimum is used as a noun, as in (i).
}

(i) I need a minimum of 300 points to win the bet. 
What such expressions appear to have in common is that they operate on existential structures. To be more precise, operators like superlatives and minimum/maximum select the top-most or bottom-most element from a scale. In examples like (25), no scale is available: the number of people invited by John is a definite value (a singleton set):

$$
\lambda n . J o h n \text { invited (exactly) } n \text { people }
$$

Applying minimally to (27) makes no sense, since this set contains just the single value. In order to make the application of the end-point operator meaningful, a scale needs to be created. This can be done by assuming that there is a silent existential quantifier somewhere in the embedded structure. For instance, the following would result in a scale for which the application of an end-point operator does make sense.

$$
\lambda n \text {.John may have invited (exactly) } n \text { people }
$$

Note that we can observe something similar with superlatives. The amount of prize money won by Federer is in principle a definite amount. However, in the scope of a superlative, we need to divide this amount up into parts.

(29) The highest amount of prize money that Federer won so far is $\$ 1$ million. That was for Wimbledon 2009.

the amount of prize money that Federer won in some tournament

The highest amount of prize money that Federer won so far is $\$ 8.3$ million. That was over 2006.

the amount of prize money that Federer won for some year

When we interpret (29) or (30) we need to assume that the speaker had some silent indefinite in mind in the scope of the superlative. Without such an assumption, interpretation will fail, for no scale will have been created.

What the puzzles of minimum and maximum requirement have in common with examples like $(29) /(30)$ is then the presence of an operator that requires a certain scale structure. When the overt material does not lead to an interpretation in which the operator is applied to a scale, the insertion of some existential quantificational structure can be used to 'save' interpretation. In the case of minimum and maximum requirements, this last-resort strategy involves local existential closure over the function selecting the relevant part of the modal base.

\section{To conclude}

The main goal of this paper is to point out that the semantics of minimum (and maximum) requirements is not trivial. The analysis I have sketched is open for improvement, but, importantly, I hope to have shown that there is a 
connection between the puzzles I discussed here and the recent literature on modified numerals.

I have remained silent about a further possible connection. As I mentioned in the previous section, an operator like minimum has a kinship to the relation $\geq$, which in turn is linked to disjunction: $x \geq y \Leftrightarrow x=y \vee x>y$. To do minimally $p$ means to do $p$ or to do more than $p$. With this in mind, the puzzles I have discussed above are yet further additions to the list of puzzles that involve modality and disjunctive structures, most notably the issue of free choice possibility. However, I will need to leave a detailed comparison to free choice for further research. The analysis I proposed is based on a general observation I made concerning scalar end-point markers, namely that since their complement needs to denote a scale, their presence sometimes leads to the insertion of a covert existential quantifier to facilitate interpretation. Although the analysis incorporates elements familiar from the free choice literature (such as Klinedinst's view on modality), the main mechanism seems to me to be based on an independent observation.

Acknowledgments. This work was supported by a grant from the Netherlands Organisation for Scientific Research (NWO), which I hereby gratefully acknowledge. I would like to thank an anonymous Amsterdam Colloquium reviewer for several comments, as well as David Beaver, Jakub Dotlacil, Donka Farkas, Janneke Huitink, Dan Lassiter, Jessica Rett, Philippe Schlenker, Magda Schwager and Benjamin Spector for discussing the issues in this paper with me.

\section{References}

1. Kai von Fintel and Sabine Iatridou. Epistemic containment. Linguistic Inquiry, 34(2):173-198, 2003.

2. Kai von Fintel and Sabine Iatridou. What to do if you want to go to harlem: Anankastic conditionals and related matters. Ms. MIT, http://mit.edu/fintel/www/harlem-rutgers.pdf, 2005.

3. Bart Geurts and Rick Nouwen. At least et al.: the semantics of scalar modifiers. Language, 83(3):533-559, 2007.

4. Nathan Klinedinst. Plurality and Possibility. PhD thesis, University of California Los Angeles, 2007.

5. Manfred Krifka. More on the difference between more than two and at least three. Paper presented at University of California at Santa Cruz, 2007.

6. Rick Nouwen. Two kinds of modified numerals. Semantics and Pragmatics, 3(3):141, 2010.

7. Hotze Rullmann, Lisa Matthewson, and Henry Davis. Modals as distributive indefinites. Natural Language Semantics, 16:317357, 2008.

8. Magdalena Schwager. Exhaustive imperatives. In Paul Dekker and Michael Franke, editors, Proceedings of the 15th Amsterdam Colloquium. Universiteit van Amsterdam, 2005. 